\title{
Portfolio performance under tracking error and asset weight constraints
}

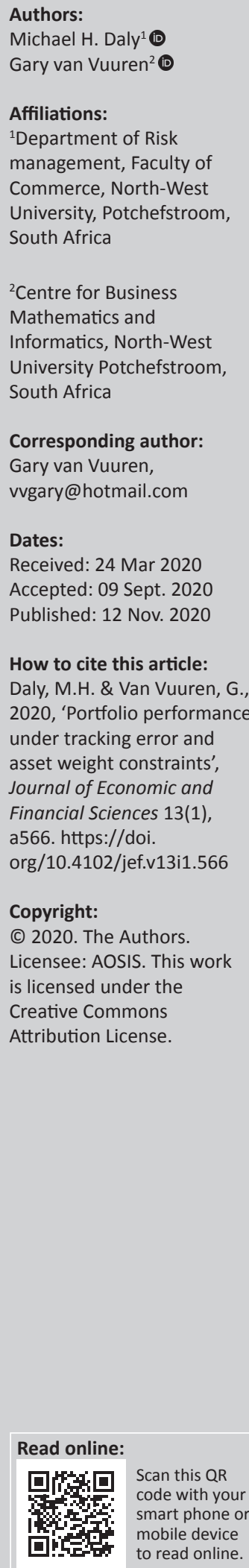

Orientation: Active portfolio managers must simultaneously maximise excess returns (over benchmarks), limit risk and observe constraints on, for example, tracking errors (TRs), betas and asset weights.

Research purpose: Determining the range of possible risk and returns attainable by such constrained portfolios is of interest to active portfolio managers. Weight restrictions reduce the range of achievable returns. This work demonstrates the magnitude of these reductions.

Motivation for the study: This research installs and augments an approach that ascertains the effect on a TR (active) constrained portfolio in absolute risk-return space. The effects are displayed in risk-return space, demonstrating the impact on such constraints.

Research approach/design and method: A theoretical approach to plot the constant TR frontier was used. Theoretical and quantitative analytical approaches to establish changes in the constant TR frontier on a simulated (but highly stylistic) market portfolios were also employed.

Main findings: Considerable reduction is observed in possible investable portfolios, even for limited asset weight restrictions. This effect is amplified if multiple restrictions are imposed simultaneously, driven by both a reduced area in risk-return space enclosed by the constant TR frontier and changes in the frontier long-axis slope.

Practical/managerial implications: The change in the long-axis slope sign is also a feature of changing economic conditions, thereby acting as an early warning signal with associated ramifications for asset managers.

Contribution/value add: The combined effects on active portfolio performance of TR and asset weight constraints have not been investigated and demonstrated before.

Keywords: active management; tracking error; weight constraints; benchmarks; market portfolios

\section{Introduction}

\section{Orientation}

Passive investment attempts to match the performance of certain market indices rather than attempting to outperform these through specific stock assessment and selection. Active investment managers invest in funds whose constituents' worth are independently assessed, whereas their passive counterparts construct portfolios of market indices in the proportion they are held in the specific index and rebalance these proportions as the market changes.

The goal of active management is to beat the market or outperform agency-determined benchmarks. These benchmarks are often inefficient, comprising assets with arbitrarily defined upper and lower limits as a result of partial securitisation and free float restrictions. Active investing is generally costlier than passive investing (research analysts and portfolio managers require compensation, and frequent trading also incurs costs), and many active managers do not beat the index after expenses are accounted for. Active managers must also comply with strict tracking error (TE) (the variance of the difference between portfolio and benchmark returns) ceilings, where punitive penalties for non-compliance can be severe (Riccetti 2012). For these reasons, passive investing often outperforms active investments.

The competition between advocates of active and passive management has, however, recently (2018) intensified. Historically, passive investing has taken preference globally; however, recent evidence identifies a change in this trend (Torr 2018). Today's (2018) narrow bull market has a 
fundamental weakness of being top heavy, with the 'big five' (Facebook, Apple, Amazon, Microsoft and Google) S\&P 500 companies driving the large US index-tracking market. These high concentration levels reduce diversification, and the emphasis on tracking the S\&P 500 has led to overcrowding of the Exchange Traded Fund (ETF) market (Brenchley 2018). A reversal of this tide would provide an opportunity for real dominance by active managers (Gilreath 2017).

The success of active portfolio managers is measured relative to a benchmark. The components of these benchmarks are frequently defined by agents who may know little about asset allocation and whose principal interest is often to limit risk through the imposition of strict weight allocation ranges. Asset allocation weights can depend on tax considerations, geographical restrictions (such as foreign exchange limits) or simply agent preferences. Active portfolio components can be under- or over-weighted relative to the benchmark, but the overall weights can also be negative, that is short positions. These constraints coupled with strict TE limits generally inhibit fund manager performance.

\section{Research purpose and objectives}

This article contributes by investigating the effect of imposing long-only portfolio component weights on active portfolios subject to TE constraints. In addition, the effect of asset weight ranges on such portfolios is also explored and new constrained frontiers are established as a result. Properties of such frontiers are of considerable interest to active fund managers and investors.

The remainder of this article proceeds as follows: the section 'Literature review' explores the relevant literature governing efficient frontiers subject to no constraints, TE frontiers which describe portfolios achieving maximal returns whilst subject to a TE constraint, constant TE frontiers which embrace all portfolios subject to various TEs and finally constrained constant TE frontiers subject to a specific TE limit and various asset weight range constraints. The section 'Research design' describes the stylised data used for the study and defines the mathematics relevant to the exposition. The results are displayed and discussed in the 'Results and discussion' section and the section 'Conclusion' concludes.

\section{Literature review}

For the fund to outperform the benchmark, the generation of a positively expected TE is implied (Roll 1992:17). An important criterion for evaluating active manager performance is minimising the TE (the standard deviation of the difference between fund and benchmark returns). Roll (1992) explored this criterion and found that funds with the smallest total return volatility implied that TEconstrained funds would pursue portfolios with a minimum TE (the former for a given expected total return and the latter for a given expected performance measured relative to a benchmark). This approach neglects absolute portfolio risk, which means these portfolios are not optimal (in mean-variance space) and they are riskier than the benchmark.

Roll (1992:19) also set out the fundamentals for establishing the TE frontier: a frontier comprising maximum excess returns (relative to a benchmark) and associated risk coordinates for different levels of TE. Jorion (2003:78) expanded on Roll's (1992:19) work and set out the relevant mathematics to construct a constant TE frontier, that is a locus of points comprising all returns and associated risk for different levels of TE. This locus was found to be an ellipse in mean-variance space and a distorted ellipse in mean-risk space. Jorion (2003) also proposed that an optimal measure of TE-constrained portfolio performance should be the return on the constant TE frontier at a risk equal to that of the benchmark. The tilt of the constant TE frontier meant that the difference between the maximum return at a given TE level and the return associated with Jorion's (2003) suggestion was relatively minor.

Maxwell et al. (2018:5848) extended Jorion's (2003) work and established the analytical solution for the optimal Sharpe ratio portfolio on the constant TE frontier. This portfolio is the TE-constrained analogue of the optimal (efficient) portfolio on the standard efficient frontier. Daly, Maxwell and Van Vuuren (2018) explored $\beta, \alpha$ and utility on constant TE frontier. The $\beta$ frontier is a parabola in riskreturn space, which defines feasible portfolios subject to a specified $\beta$. An $\alpha$-TE frontier is assembled from portfolios that have a specified $\alpha$ for a maximum TE. Utility and associated risk aversion were also explored for TEconstrained portfolios.

Maxwell and Van Vuuren (2019) investigated the behaviour of various portfolio strategies (maximally diversified, exhibit risk parity, have minimal intra-correlation and minimum risk) on the constant TE frontier. Such portfolios were found to behave differently to those which form part of the efficient set. Evans and Van Vuuren (2018) used several performance indicators to evaluate the benchmark outperformance of six active portfolio strategies - subject to a TE constraint - on the constant TE frontier.

This catalogue of work involving TE-constrained portfolios - although comprehensive - ignores a fundamental reality of active portfolios: mandated constraints on constituent asset weights. Work that covers this important aspect of active portfolios is limited.

Ammann and Zimmermann (2001:40) investigated the relation between statistical TE measures and asset allocation restrictions expressed as admissible weight ranges by simulating investment strategies subject to such constraints. By using market data, Ammann and Zimmermann (2001) found that imposing large tactical asset allocation ranges implied surprisingly small TEs. More recently, BajeuxBesnainou et al. (2011) considered portfolio performance under simultaneous TE and weight constraints compliance. 
Both equality and inequality weights constraints were considered, and the analytical and geometrical solutions for both cases were derived.

In this article, we use a stylised example of realistic market data like that employed by Bajeux-Besnainou et al. (2011) and explore the shape of the constant TE frontier under various weight constraints for different TEs. We also examine the Sharpe ratio and information ratio (IR) profiles of portfolios subject to these constraints for the first time.

\section{Research design \\ Research approach}

To establish the methodologies required for various frontiers, some definitions are first required. This section proceeds by introducing and describing the relevant variables and algebraic components. The mathematics governing the generation of the efficient frontier is then set out, followed by the algebra that defines the TE frontier and then the constant TE frontier.

\section{Research method}

Active fund managers are tasked with outperforming specified benchmarks, and the active asset positions they take may or may not be benchmark components (depending on the mandate governing the fund). The algebra required to derive the relevant investment strategy weights uses the same underlying variables, matrices and matrix notation, as defined below.

$q$ : the vector of benchmark weights for a sample of $N$ assets

$x$ : $\quad$ the vector of deviations from the benchmark

$q_{p}(=q+x):$ the vector of portfolio weights

$E: \quad$ the vector of expected returns

$\sigma: \quad$ the vector of benchmark component volatilities

$r: \quad$ the benchmark correlation matrix

$V: \quad$ the covariance matrix of asset returns and

$r_{f}: \quad$ the risk-free rate.

Net short sales are allowed in this formulation, so the total active weight $\boldsymbol{q}_{\boldsymbol{i}}+\boldsymbol{x}_{\boldsymbol{i}}$ may be negative for any individual asset, $i$. The universe of assets can generally exceed the components of the benchmark, but for Roll's (1992) methodology, assets in the benchmark must be included.

Expected returns and variances are expressed in matrix notation as:

$\mu_{B}=q^{\prime} E: \quad$ the expected benchmark return

$\sigma_{B}^{2}=q^{\prime} V q$ : the variance of benchmark return

$\mu_{\varepsilon}=x^{\prime} E$ : the expected excess return and

$\sigma_{\varepsilon}^{2}=x^{\prime} V x$ : the TE variance (i.e. TE2).

The active portfolio expected return and variance is given by $\mu_{P}=(\boldsymbol{q}+\boldsymbol{x})^{\prime} \boldsymbol{E}=\mu_{B}+\mu_{\varepsilon}$ and $\sigma_{P}^{2}=(\boldsymbol{q}+\boldsymbol{x})^{\prime} \boldsymbol{V}(\boldsymbol{q}+\boldsymbol{x})$, respectively. The portfolio must be fully invested, so $(q+x)^{\prime}$ $1=1$ where 1 represents an $\mathrm{N}$-dimensional vector of $1 \mathrm{~s}$.

Merton (1972) defined $a=\boldsymbol{E}^{\prime} \boldsymbol{V}^{-\mathbf{1}} E, b=E^{\prime} V^{-\mathbf{1}} 1, c=\mathbf{1}^{\prime} V^{-\mathbf{1}}$ $1, d=a-\frac{b^{2}}{c}$ and $\Delta_{1}=\mu_{B}-\frac{b}{c}$ where $b / c=\mu_{M V}$ and $\Delta_{2}=\sigma_{B}^{2}-\frac{1}{c}$ with $1 / c=\sigma_{M V}^{2}$ where $M V$ is the minimum variance portfolio.

Note that deviations from the benchmark are represented by $\boldsymbol{x}$, the total portfolio component weights are $q+x\left(=q_{p}\right)$ and where the investment strategy is unaffected by TE constraints, the relevant portfolio weights, $w$, are used.

\section{Mean variance frontier in risk-return space}

Minimise $q_{P}^{\prime} V q_{P}$ subject to $q_{P}^{\prime} 1=1$ and $q_{P}^{\prime} E=G$ where $G$ is the target return. The vector of portfolio weights is $q_{P}=\left(\frac{a-b G}{d}\right) q_{M V}+\left(\frac{b G-\frac{b^{2}}{c}}{d}\right) q_{T G}$ where $q_{M W}$ is the vector of asset weights for the minimum variance portfolio given by $q_{M V}=V^{-1} \frac{1}{c}$ and $q_{T G}$ is the vector of asset weights for the

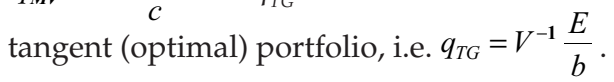

The locus of points in return-risk space is the efficient frontier, not subject to sales constraints, that is short-selling of assets is permitted (as shown in Figure 1). Imposing a long-only constraint on efficient portfolios requires a recursive algorithm to solve for constituent weights, there is no closedform solution to this problem.

\section{Unconstrained tracking error frontier in risk-return space}

Maximise $x^{\prime} E$ subject to $x^{\prime} 1=0$ and $\boldsymbol{x}^{\prime} \boldsymbol{V} \boldsymbol{x}=\sigma_{\varepsilon}^{2}$. The solution for the vector of deviations from the benchmark is $x= \pm \sqrt{\frac{\sigma_{\varepsilon}^{2}}{d}} V^{-1}\left(E-\frac{b}{c} 1\right)$. The solution to this optimisation problem generates the TE frontier, a portfolio's maximal return at a given risk level and subject to a TE constraint (as shown in Figure 1).

The benchmark - also indicated in Figure 1 - may be efficient, in which case it would lie on the efficient frontier.

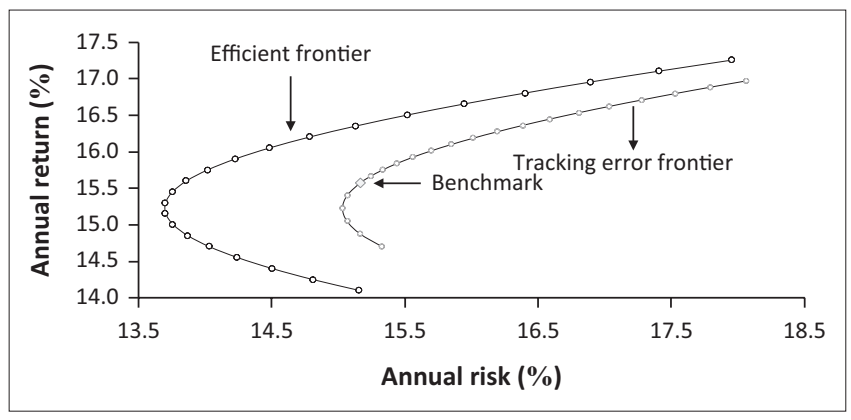

FIGURE 1: Efficient frontier and tracking error frontier in mean-standard deviation space. The diamond marker indicates the benchmark on the tracking error frontier. 
In reality, the benchmark is somewhat arbitrarily selected (a mix of stock and bonds or an inefficient market index), so it is frequently not a member of the efficient portfolio set. This TE frontier passes through the benchmark position where $T E=0$.

\section{Unconstrained constant tracking error frontier}

Maximise $x^{\prime} E \quad$ subject to $x^{\prime} 1=0, \quad \boldsymbol{x}^{\prime} \boldsymbol{V} \boldsymbol{x}=\sigma_{\varepsilon}^{2} \quad$ and $(q+x)^{\prime} \boldsymbol{V}(q+x)=\sigma_{P}^{2}$.

The vector of deviations from the benchmark is $x=-\frac{1}{\lambda_{2}+\lambda_{3}} V^{-1}\left(E+\lambda_{1}+\lambda_{3} V q\right) \quad$ where $\quad \lambda_{1}=-\frac{\lambda_{3}+b}{c}$, $\lambda_{2}= \pm(-2) \sqrt{\frac{d \Delta_{2}-\Delta_{1}^{2}}{4 \sigma_{\varepsilon}^{2} \Delta_{2}-y^{2}}}-\lambda_{3}$ and $\lambda_{3}=-\frac{\Delta_{1}}{\Delta_{2}} \pm \frac{y}{\Delta_{2}} \sqrt{\frac{d \Delta_{2}-\Delta_{1}^{2}}{4 \sigma_{\varepsilon}^{2} \Delta_{2}-y^{2}}}$. Jorion (2003:81) established that the solution for this optimisation describes an ellipse - a constant TE frontier - in return-risk space: the unconstrained ${ }^{1}$ constant TE frontier (Figure 1). This frontier establishes the boundary of possible risk-return combinations of (i.e. satisfies the mandatory TE constraints) active portfolios. The upper segment of this frontier, shown in Figure 2, is bounded on the left by the minimum variance portfolio and above by the maximum return portfolio. The arc between these portfolios on the unconstrained constant TE frontier represents the efficient set of portfolios subject to a specific TE, in this example 5\% (Palomba \& Riccetti2019).

We display the entire TE ellipse to demonstrate the domain of all possible TE-constrained portfolios, even the inefficient ones. In our experience, although portfolio managers do not consider portfolios with high risk, given the same return, sometimes the vagaries of market conditions render their portfolios inefficient. Note that each risk-return combination within a TE ellipse (even the benchmark risk and return) has the same TE. The benchmark's risk-return combination may be achieved via many different constituent weight combinations - not just the unique benchmark configuration. If the benchmark's risk-return coordinate is within the TE ellipse, a combination of constituent weights exists, which leads to the same risk-return benchmark coordinate. The asset weights will nevertheless be sufficiently different from the benchmark weights to warrant a $T E \neq 0$.

The locus of the constant TE ellipse in return-risk space under increasing TEs is informative.

At first localised on the benchmark portfolio position, the ellipse expands (long and short axes increase) as TE increases until the left end coincides with the efficient frontier's turning point region. Increasing TE still further drags the ellipse back to the right and increasing TE further shifts the frontier so far to the right that the benchmark is eventually excluded, that is 1.Unconstrained because there are no restrictions on the values of the relative asset weights, $x$.

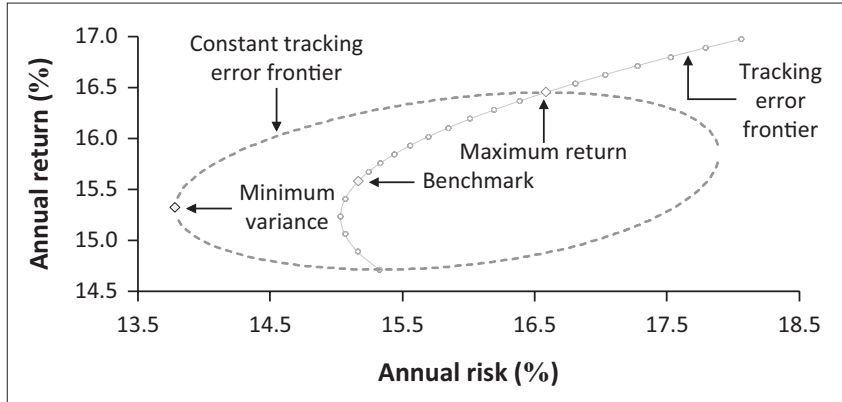

FIGURE 2: Constant (unconstrained) tracking error frontier for $T E=5 \%$ and $\mathrm{TE}$ frontier for $0 \% \leq T E \leq 8 \% .2$

TABLE 1: Stylised input data.

\begin{tabular}{|c|c|c|c|c|c|c|c|}
\hline \multirow[t]{2}{*}{ Assets } & \multicolumn{5}{|c|}{ Domestic } & \multicolumn{2}{|c|}{ Foreign } \\
\hline & $d_{1}$ & $d_{2}$ & $d_{3}$ & $d_{4}$ & $d_{5}$ & $d_{1}$ & $d_{2}$ \\
\hline Benchmark weights (\%) & 14.3 & 14.3 & 14.3 & 14.3 & 14.3 & 14.3 & 14.3 \\
\hline Annual return $(\%)$ & 16.7 & 16.0 & 14.9 & 15.8 & 14.5 & 12.0 & 15.3 \\
\hline Annual volatility (\%) & 19.0 & 27.0 & 22.0 & 26.0 & 21.0 & 27.0 & 26.0 \\
\hline \multirow[t]{7}{*}{ Correlation matrix } & 1 & 0.3 & 0.3 & 0.3 & 0.3 & 0.2 & 0.2 \\
\hline & 0.3 & 1 & 0.3 & 0.3 & 0.3 & 0.2 & 0.2 \\
\hline & 0.3 & 0.3 & 1 & 0.3 & 0.3 & 0.2 & 0.2 \\
\hline & 0.3 & 0.3 & 0.3 & 1 & 0.3 & 0.2 & 0.2 \\
\hline & 0.3 & 0.3 & 0.3 & 0.3 & 1 & 0.2 & 0.2 \\
\hline & 0.2 & 0.2 & 0.2 & 0.2 & 0.2 & 1 & 0.2 \\
\hline & 0.2 & 0.2 & 0.2 & 0.2 & 0.2 & 0.2 & 1 \\
\hline
\end{tabular}

it lies outside the ellipse. In such cases, the TE is sufficiently loose to permit a high enough level of risk between the portfolio and the benchmark as to exclude benchmark constituents entirely.

\section{Long-only, inequality weight-constrained constant tracking error frontier}

Here we use the results of Bajeux-Besnainou et al. (2011), allowing $\omega \geq 0$ or $\leq 0$ (i.e. short positions permitted), and consider a subset $l$ of the $N$ traded assets. These are the limited or restricted assets because portfolios will be constrained to a limited weight or limited range of weights in these $l$ assets. Then $1_{l}$ is the $N$-dimensional vector such that:

$\mathbf{1}_{\ell}=\left\{\begin{array}{l}1 \text { if } \text { asset }_{i} \in \ell \\ 0{\text { if } \text { asset }_{i} \notin \ell}\end{array}\right.$

Consider an inequality constraint $\mathbf{1}^{\prime} \boldsymbol{q}_{\boldsymbol{P}} \leq \omega$ where $\omega$ is a weight constraint such that the weights may be $\geq 0$ or $\leq 0$ provided $\sum \omega_{i}=1$. An example shown in Table 1 might be that the sum of domestic asset weights must not constitute more than half the portfolio or:

$\sum_{i=1}^{\mathrm{N}} \omega_{d_{i}} \leq 50 \%$

2.The TE frontier is the locus of risk-maximum return points for many different TEs. The constant TE frontier is the locus of points, which encloses all possible riskreturn combinations for a single TE. The maximum return obtainable for a given TE return combinations for a single TE. The maximum return obtainable for a given TE
on the constant TE frontier is, by definition, also a point on the TE frontier-hence their intersection at the maximum return. 
Bajeux-Besnainou et al. (2011) define a risk-aversion parameter, $\gamma$, and a risk-tolerance parameter, $\theta$, which are related by:

$$
\theta=\frac{1^{\prime} V^{-1} E}{\gamma}
$$

The objective is to maximise $\boldsymbol{E}^{\prime} \boldsymbol{x}-\frac{\gamma}{2} \boldsymbol{x}^{\prime} \boldsymbol{V} \boldsymbol{x}$ (recall that $\boldsymbol{x}=\boldsymbol{q}_{\boldsymbol{P}}-\boldsymbol{q}$, i.e. deviations from the benchmark) subject to $\mathbf{1}^{\prime} \boldsymbol{x}=\mathbf{0}$ and $\mathbf{1}_{\ell}^{\prime} \boldsymbol{q}_{\boldsymbol{P}} \leq \omega$. Bajeux-Besnainou et al. (2011) provide derivations and solutions for this and other formulations.

The loci of the inequality weight-constrained constant TE frontier - as constraints change - are shown in Figure 3, and the consequences of the changing shape are discussed in the section 'Results and discussion'.

\section{Measuring instruments}

The data comprised simulated realistic weights, returns, volatilities and correlations for a small, standardised benchmark comprising equal weights in seven assets (five domestic: $d_{1}$ to $d_{5^{\prime}}$ and two foreign: $f_{1}$ and $f_{2}$ ) with the stylised description as given in Table 1. This numerical example - and the reason for using simulated rather than historical parameters - is to provide continuity and to allow for better comparison of our results with those obtained by Bajeux-Besnainou et al. (2011).

\section{Research procedure}

We also tested portfolios comprising assets with higher correlations, with correlations $<0$ and using some negative expected returns. The results were broadly the same, even though some of the calculated weights for the optimisation procedure become negative as a consequence of these changes.

Portfolio constituents were derived from the universe of investable assets (i.e. domestic and foreign components).
Note that the assets which constitute the portfolio in the following examples could be asset classes (such as equity, bonds and cash), specific industry sectors within an asset class (e.g. an industrial equity index, a banking and finance index, etc.) or individual assets such as single name stocks or bonds.

\section{Ethical consideration}

This article followed all ethical standards for research without direct contact with human or animal subjects.

\section{Results and discussion}

By using the stylised example set out in the section 'Research design', comprising domestic and foreign assets (Table 1), various constraints were placed on portfolio constituents to explore their effects on the shape of the constant TE frontier.

A common restriction on component weights is that longonly positions are permitted, i.e. $q_{P}>0$. Individual assets may still be underweight in relation to the benchmark, in which case $x<0$, but $q+x>0$. Imposing this restriction shrinks the ellipse of feasible portfolios, where similar returns for low levels of risk can be achieved (i.e. the southwest end of the ellipse). However, as more risk is taken on, a greater deviation from the unconstrained constant TE frontier can be observed (eastern end). The east and north ends of the ellipse contract (i.e. both the long [south-west to north-east] and short [north-west to south-east] axes shorten) and the slope of the long axis decreases. Recent research (Gunning \& Van Vuuren 2019) has also established that slope sign changes can act as early warning signals for the onset of economic downturns with significant ramifications for fund managers.

Some results are presented in Figure 3 - all for a constant $T E=5 \%$.

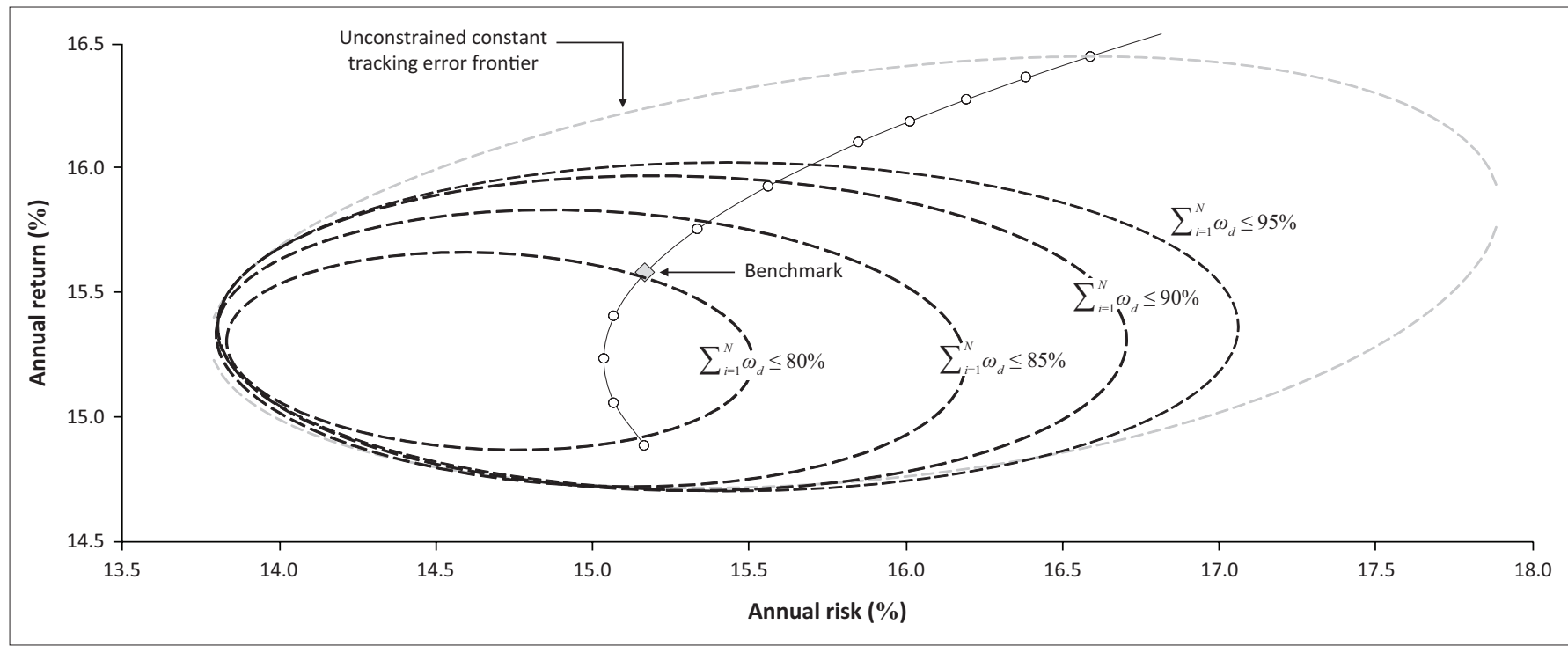

FIGURE 3: Unconstrained (long and short positions permissible) constant tracking error frontier for $T E=5 \%$ and the sum of domestic weights $\sum_{i} w_{d i} \leq 95 \%, 90 \%, 85 \%$ and $80 \%$. 


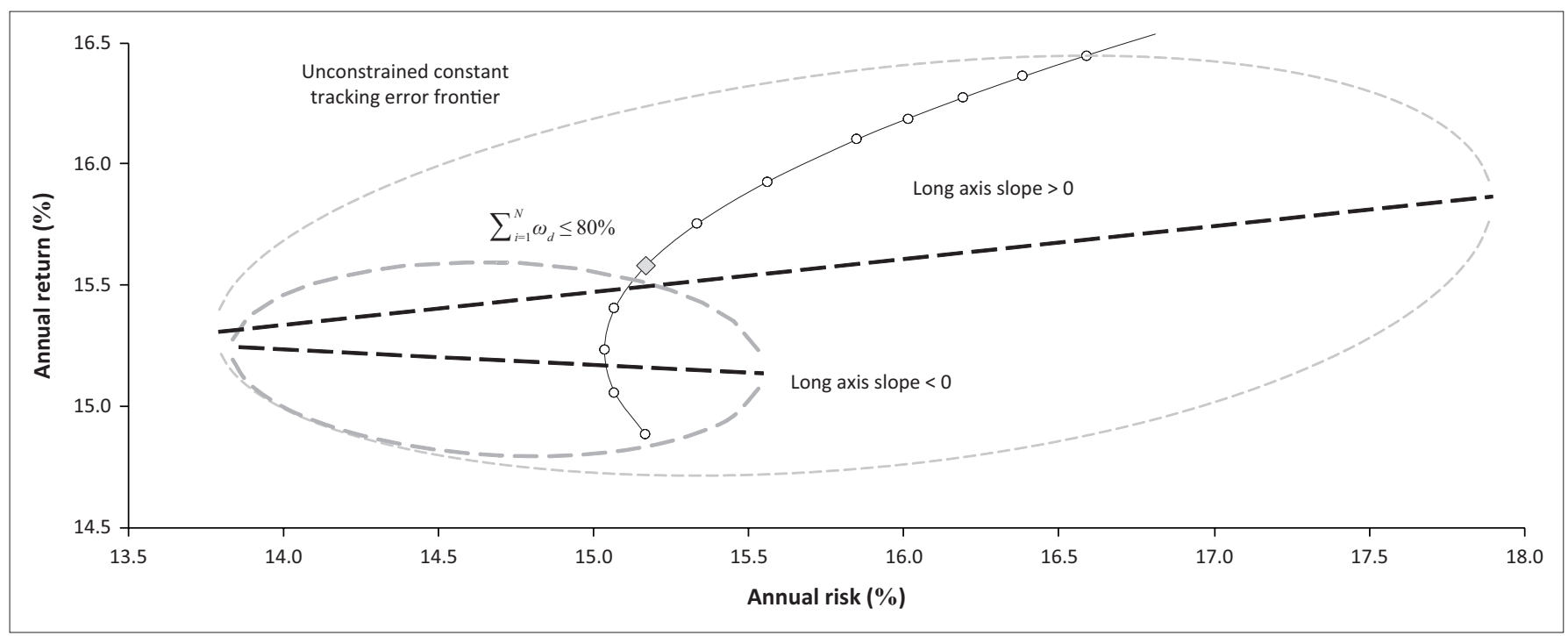

FIGURE 4: Sign of slopes of long axes for unconstrained constant $T E=5 \%$ frontier and $T E=5 \%$ frontier with domestic weights constrained to $\leq 80 \%$.

The grey dashed line shows the unconstrained constant TE frontier. This is the locus of points in return-risk space defined by the maximum and minimum obtainable annual returns at each level of risk subject to a portfolio TE constraint only (i.e. weights can be positive or negative).

Imposing the inequality $\sum_{i=1}^{N} \omega_{d_{i}} \leq \omega$ (for decreasing $w \mathrm{~s}$ ) continues to shrink both long and short axes, with the result that the original ellipse 'deflates' whilst remaining hinged to the south-west end. As $\sum_{i=1}^{N} \omega_{d_{i}}$ recedes from $100 \%$, the portfolio's maximum returns - even for low $\sigma_{P} \mathrm{~s}$ - decrease. At $\sum_{i=1}^{N} \omega_{d_{i}} \leq 80 \%$, the benchmark already falls outside the frontier. For a TE $=5 \%$ and an inequality restriction that the sum of the domestic weights must be $<80 \%$, the benchmark falls outside the ellipse (in this stylised example), meaning that the benchmark's unique combination of risk and return is not possible for a portfolio subject to these (relatively mild) constraints. The important point is that - even for relatively moderate limits on acceptable asset weights - the range of portfolios that satisfy the relevant constraints becomes severely diminished, and even the benchmark portfolio is rendered unattainable, a result also obtained by Wagner (2003) and Palomba and Riccetti (2012).

The slope of the ellipse's long axis also decreases at the asset weight restrictions increase. For the unconstrained constant $\mathrm{TE}$, the slope is positive (and $\approx 0.13$ ) for $T E=5 \%$ as shown in Figure 4 for the unconstrained constant TE frontier (showing a positive long-axis slope) and the constant TE frontier where $\sum_{i=1}^{N} \omega_{d_{i}} \leq 80 \%$ (showing a negative long-axis slope).

For increasing constraints on $\sum_{i=1}^{N} \omega_{d_{i}}$, the slope diminishes but less rapidly as the constraint increases. At the relatively benign constraint of $\sum_{i=1}^{N} \omega_{d_{i}} \leq 88 \%$, the long-axis slope becomes negative. This change in the constant TE's long-axis slope has important consequences for asset allocation and managing investment styles. Even mild constraints (in the sense that this restriction is common in active portfolio management and often more so than this) change the slope sign and result in inefficient portfolios (see Gunning \& Van Vuuren 2019).

The inclusion of a risk-free asset in the portfolio was also considered. Inverting a variance covariance matrix (VCV) with one (or more) component $=0 \%$, however, results in divisions by 0 and associated intractable mathematical problems, so a small - but non-zero risk - 'risk free' security was included. ${ }^{3}$ This addition did not alter the results - the position of the ellipse in return-risk space was altered, but the orientation of the ellipse's axes and the reduction in area of possible portfolios were negligible unless the weight of the risk-free security was unrealistically high.

Allowing for short selling did not change the results either. When the weight restrictions were imposed, portfolio weights were always $>0 \%$ for the configuration employed in this work (i.e. volatilities, expected returns, correlations and benchmark weights) (see Figure 3). For correlations $<0$ or for negative expected returns, weights $<0 \%$ become relevant as the benefits from diversification are invoked to maximise portfolio returns. Again, however, this did not change the orientation of the ellipse's axes nor the rate of reduction in investable portfolio area.

We also considered multiple constraints, for example, the imposition of weight restrictions on both domestic and foreign securities simultaneously. The universe of possible portfolios shrinks faster - with increasing restrictions - than that observed for restrictions on only domestic assets. Portfolio constraints in the form of weight restrictions reduce the portfolio choice considerably to the point of vanishing relevance as these constraints become more limiting as shown in Figure 5.

Imposing weights constraints reduces the number of investable portfolios because of an overall reduction in TE 3.Such a security is not unrealistic. 


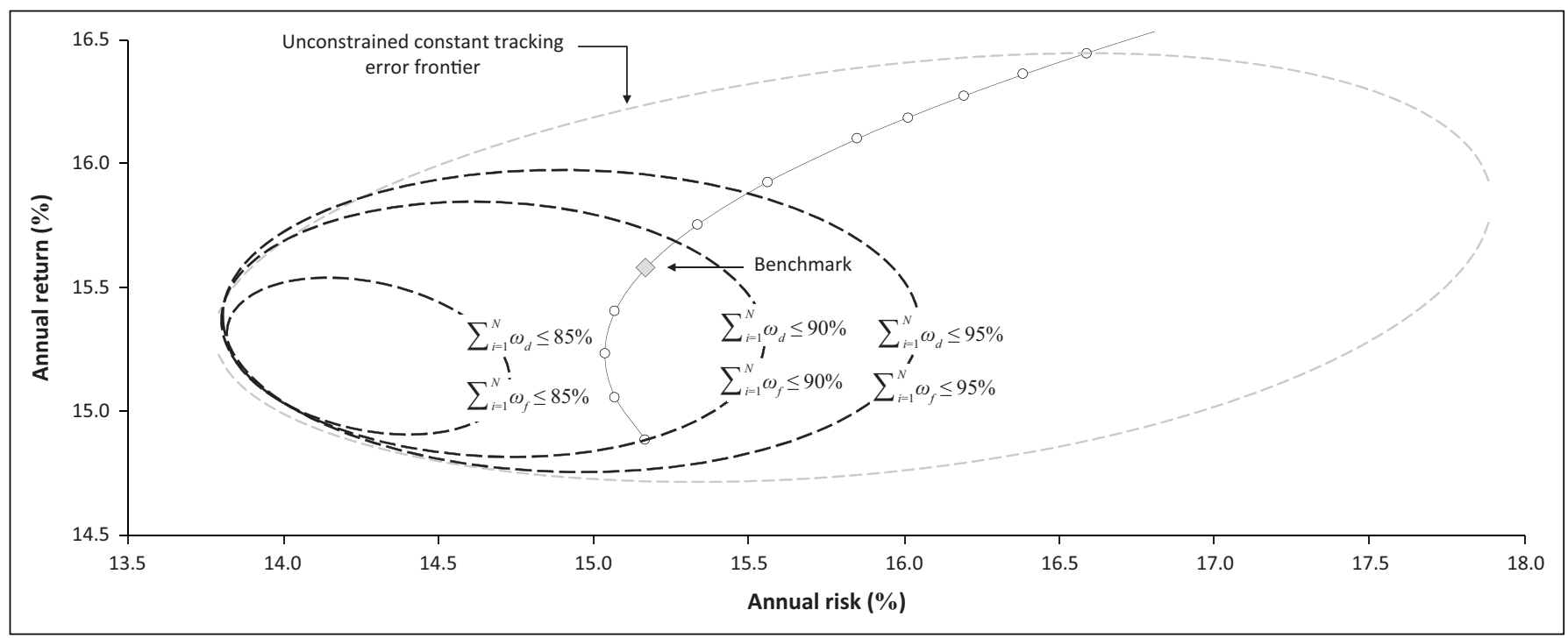

FIGURE 5: Unconstrained (long and short positions permissible) constant tracking error frontier for $T E=5 \%$, sum of domestic weights $\sum w_{d i} \leq 95 \%$ and $90 \%$ and sum of foreign weights $\sum_{i} w_{f i} \leq 95 \%, 90 \%$. Note that, to satisfy these constraints, investment in a 'risk-free' asset becomes necessary.

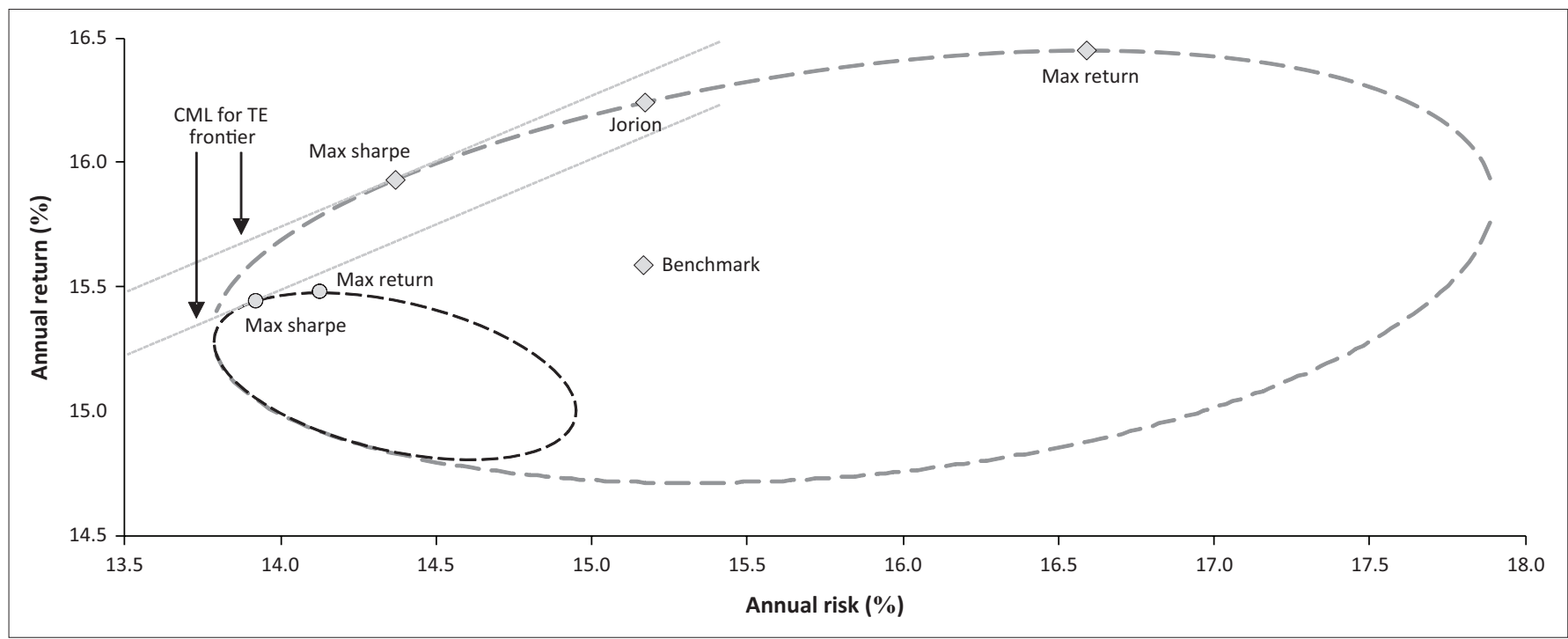

FIGURE 6: Impact of weight constraints on investable portfolios. Note the truncated axes.

ellipse region as well as a telescoped efficient arc brought about by a negative long-axis slope (Figure 6). Maximum Sharpe ratio portfolios occur where the 'capital market line' (originating on the return axis at the risk-free rate) is tangent to the constant TE frontiers. For larger constant TE ellipses (unconstrained), the long-axis slope is positive, and the range of portfolio efficiency spans a wide range of portfolio risk. For smaller constant TE ellipses, i.e. ones for which a weight constraint has been imposed, not only has the TE ellipse shrunk considerably but also the change in the long-axis slope from positive to negative has occurred, thereby reducing the efficient arc 'length'. The combined effect of these TE ellipse changes substantially reduces potential investment possibilities.

The Sharpe ratio was calculated for increasing domestic asset weight restrictions between $80 \% \leq w_{d} 100 \%$ on the constrained constant TE frontier, given a constant TE of $5 \%$. The constraints precipitate an imploding investible universe, where annual returns of a portfolio and subsequently the return of the maximum Sharpe ratio portfolio exponentially decrease, for each feasible risk exposure as shown in Figure 7.

It is interesting to note that, although the range of attainable Sharpe ratios diminishes with increasing domestic asset weight constraints, the maximum Sharpe ratio for each constrained portfolio does not change much. This is because the north-west corners of these ellipses are hinged - which coincide with the position of the maximum Sharpe ratio because of the strong negative long-axis slope.

The IR is given by:

$$
I R=\frac{\text { Excess return }}{T E}
$$

where 'excess return' is the return in excess of the benchmark return. 


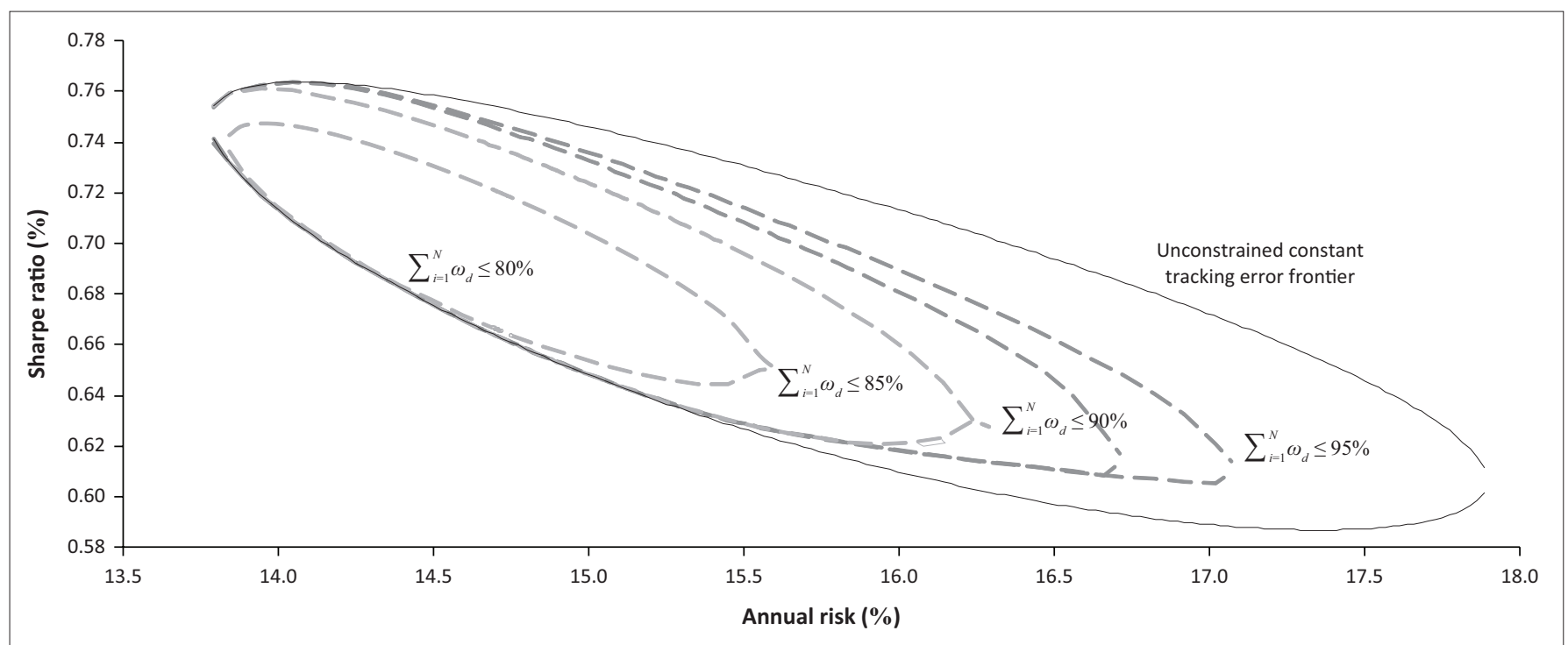

FIGURE 7: Sharpe ratios versus annual portfolio risk for $T E=5 \%$ and $\sum_{i} \omega_{d i} \leq 95 \%, 90 \%, 85 \%$ and $80 \%$.

Bajeux-Besnainou et al. (2011) introduced the adjusted IR (AIR), that is the IR of a portfolio subject to a weight constraint. Such a portfolio may be understood by invoking an adjusted benchmark defined as the closest portfolio to the benchmark, which satisfies the TE and other relevant constraints. The adjusted benchmark is, however, not observable and must be assembled by using empirical historical returns. For this reason, we avoided the use of the AIR.

Both IR and Sharpe ratios diminish with increasing restrictions. Unconstrained portfolios occupy the largest area in risk-return space, and this space diminishes as constraints are added. The more restrictive the constraint, the smaller the potential investment area and the lower the performance ratio attainable. The IR diminishes to zero (and can become negative for more severe constraints on asset weights) because at these high weight constraints, the excess return over the benchmark approaches $0 \%$ (see Figure 3).

\section{Conclusion}

\section{Outline}

Optimising portfolios' risk and return originated with Markowitz (1956). The literature has since seen a proliferation of research, which has evolved from the original meanvariance space to relative mean-variance space (i.e. active funds whose performance is measured relative to a benchmark). Research then followed an interrogation of TEconstrained portfolios, but to date, not much work has been conducted on, for example, TE-constrained and asset weightconstrained portfolios simultaneously. Portfolio optimisation in excess return space subject to these multiple constraints is an area, which lacks fundamental research.

\section{Practical implications}

This work - by using stylised market data for simulations developed the constant TE frontier subject to asset weight constraints and established the region in mean-variance space of possible risk-return coordinates for increasingly restrictive boundaries. Unconstrained (i.e. long and short absolute positions permitted) portfolios subject to a TE occupied the largest possible area in risk-return space. Each subsequent restriction diminished this area by shortening both the long and short axes of the constant TE ellipse. This shortening was asymmetrical: the left end of the long axis and the bottom end of the short axis were fixed for increasing restrictions on asset components - thus, these constraints reduce the maximum returns attainable whilst reducing the risk. The range of possible investable portfolios (i.e. the region in risk-return space enclosed by the constant TE frontier) shrinks rapidly and considerably with increasing severity of restrictions, even for relatively small constraints. Combining multiple constraints, such as restrictions on both domestic and foreign weights, amplifies this reduction. The change in the slope of the constant TE frontier's long axis reduces the range of investable portfolios by shrinking the range of the efficient portfolio set.

At sufficiently 'severe' constraints, the benchmark lies outside the realm of possible portfolios. This means that for suitably restrictive constraints, the region of possible riskreturn combinations does not embrace a sufficiently large area to include the benchmark. The benchmark and portfolio are considerably different, and acceptable risk-return coordinates for the former do not apply to the latter.

\section{Limitations and recommendations}

This work used stylised simulation inputs - future work could consider real portfolio data evolving over time to establish how the constant TE frontier changes in crisis periods when also constrained by asset weights.

Normal distributions of returns are assumed in the meanvariance framework; this restrictive assumption could be relaxed in ongoing studies to consider other return 
distributions, including fat-tailed ones such as Students't or Cauchy. Relative portfolio performance, under even more constraints such as those imposed by $\beta$ and $\alpha$ mandated requirements, could also be considered. Although related portfolios have been investigated (Daly et al. 2018), earlier work did not include the effect of asset weight constraints.

\section{Acknowledgements Competing interests}

The authors have declared that no competing interest exists.

\section{Authors' contributions}

Both authors contributed equally to this work.

\section{Funding information}

This research received no specific grant from any funding agency in the public, commercial or not-for-profit sectors.

\section{Data availability statement}

Data sharing is not applicable to this article as no empirical data were used - only simulated data.

\section{Disclaimer}

The views and opinions expressed in this article are those of the authors and do not necessarily reflect the official policy or position of any affiliated agency of the authors.

\section{References}

Ammann, M. \& Zimmermann, H., 2001, 'Tracking error and tactical asset allocation', Financial Analysts Journal 57(2), 32-43.
Bajeux-Besnainou, I., Belhaj, R., Maillard, D. \& Portait, R., 2011, 'Portfolio optimization under tracking error and weights constraints', The Journal of Financia Research 34(2), 295-330. https://doi.org/10.2139/ssrn.963997

Brenchley, D., 2018, 'Why it pays to back active fund management in 2018', Morningstar, viewed 21 August 2018, from https://www.morningstar.co.uk/uk/ news/165984/why-it-pays-to-back-active-fund-management-in-2018.aspx.

Daly, M., Maxwell, M. \& Van Vuuren, G., 2018, 'Feasible portfolios under tracking error, $\beta \alpha$, and utility constraints', International Journal of Finance and Economics 15(1), 141-153. https://doi.org/10.21511/imfi.15(1).2018.13

Evans, C. \& Van Vuuren, G., 2018, 'Assessing investment strategy performance under tracking error constraints', Investment Management and Financial Innovations 16(1), 239-257.

Gilreath, D., 2017, The tide has turned: Active outpacing passive investing, CNBC, p. 1, viewed 14 September 2018, from https://www.cnbc.com/2017/09/18/the-tidehas-turned-active-outpacing-passive-investing.html.

Gunning, W. \& Van Vuuren, G., 2019, 'Exploring the drivers of tracking error constrained portfolio performance', Cogent Economics \& Finance 7(1), 1-15. https://doi.org/10.1080/23322039.2019.1684181

Jorion, P., 2003, 'Portfolio optimization with tracking-error constraints', Financial Analysts Journal 59(5), 70-82. https://doi.org/10.2469/faj.v59.n5.2565

Markowitz, H., 1956, 'The optimization of a quadratic function subject to linear constraints', Naval Research Logistics Quarterly 3(1-2), 111-133. https://doi. org/10.1002/nav.3800030110

Maxwell, M., Daly, M., Thomson, D. \& Van Vuuren, G., 2018, 'Optimising tracking error-constrained portfolios', Applied Economics 50(54), 5846-5858. https://doi. org/10.1080/00036846.2018.1488069

Maxwell, M. \& Van Vuuren, G., 2019, 'Active investment strategies subject to TE constraints', International Advances in Economic Research 25(2), 309-322. https://doi.org/10.1007/s11294-019-09746-3.

Merton, R.C., 1972, 'An analytic derivation of the efficient portfolio frontier', The Journal of Financial and Quantitative Analysis, 7(4), 1851-1872. http://dx.doi. org/10.2307/2329621

Palomba, G. \& Riccetti, L., 2012, 'Portfolio frontiers with restrictions to tracking error volatility and value at risk', Journal of Banking and Finance 36(2), 2604-2615. https://doi.org/10.1016/j.jbankfin.2012.05.014

Palomba, G. \& Riccetti, L., 2019,'Asset management with TEV and VaR constraints: The constrained efficient frontiers', Studies in Economics and Finance 36(3), 492-516. https://doi.org/10.1108/SEF-09-2017-0255

Riccetti, L., 2012, 'Using tracking error volatility to check active management and fee level of investment funds', Global Business and Economics Review 14(3), 139-158. https://doi.org/10.1504/GBER.2012.047801

Roll, R., 1992, 'A mean/variance analysis of tracking error', The Journal of Portfolio Management 18(4), 13-22. https://doi.org/10.3905/jpm.1992.701922

Torr, A., 2018, 'Fortunes of active management industry look set to improve', Business Day, viewed n.d., from https://www.businesslive.co.za/bd/opinion/2018-05-17fortunes-of-active-management-industry-look-set-to-improve

Wagner, N., 2003, 'Methods of relative portfolio optimization', in S.E. Satchell \& A. Scowcroft (eds.), New advances in portfolio construction and implementation, pp. 333-341, Butterworth and Heinemann, Oxford. 